\title{
Comparison of Oral Health Promotion and Dementia Prevention Behavior according to the Perception Level on the Relationship between Them
}

\author{
Yu-Rin $\mathrm{Kim}^{\dagger}$ \\ Department of Dental Hygiene, Silla University, Busan 46958, Korea
}

\begin{abstract}
Background: The incidence of dementia in Korea is a serious social problem, as the number of patients with dementia is increasing with a decrease in the age of dementia onset dementia is associated with oral disease among various causes, but there is very low awareness of the relationship between dementia and oral health. Therefore, in view of the above, we aimed to check oral health promotion and dementia prevention behaviors and use them as basic data for preventing dementia.

Methods: In this study, we conducted a month-long survey of people aged 40 years and above living in South Korea, and reclaimed 140 survey questionnaires. Frequency analysis was performed for the perception level of the relationship between dementia and oral health, and the demographic characteristics assessed according to the perception level. Independent t-tests were performed for the mean comparison between oral health promotion and dementia prevention behaviors. Correlation analysis was performed for the relationship between the three variables (oral health promotion and dementia prevention behaviors, the perception level of the relationship between them).

Results: The results showed that groups with a high level of awareness of the relationship between dementia and oral health were more likely to develop oral health promotion and dementia prevention behaviors $(p<0.01)$. In addition, the higher the level of awareness related to dementia and oral health, the greater the likelihood of oral health and dementia prevention behaviors ( $p<$ 0.01 ).

Conclusion: Therefore, we should try to improve dementia prevention and oral health promotion by providing accurate knowledge and awareness of the relevance between dementia and oral health.
\end{abstract}

Key Words: Dementia, Health behavior, Knowledge, Oral health

\section{Introduction}

In 2019 , Korea's elderly population was $14.9 \%$ of the total $^{1)}$, and is steadily increasing (it was $14.2 \%$ in 2017$)^{2)}$. As a consequence of low birth rate and extended life expectancy in Korea the aging population is progressing so rapidly that it is difficult to find a comparison elsewhere in the world ${ }^{3)}$. In general, as age increases, a person's cognitive ability decreases, which is an early symptom of dementia and requires attention ${ }^{4)}$.

Dementia is an acquired multiple disorder that causes daily life problems, such as memory, speech, judgment, and performance deterioration due to a degenerative brain disease or cerebrovascular disease. Furthermore, the exact cause and treatment have not been identified ${ }^{5)}$. A Delphi consensus study in $2005^{5)}$ stated that there were 4.6 million new cases annually worldwide, which indicates a new diagnosis every seven seconds. According to the World Health Organization ${ }^{6}$, there were 7.7 million new patients with dementia in 2012, which is one every 4 seconds and increasing rapidly as the years go by. In Korea, the prevalence of dementia among those aged 65 
years and above was $9.0 \%$ in 2015 and $10.0 \%$ in 2018 . The proportion of patients with dementia is expected to increase by $10.29 \%$ in 2020 , and $16.09 \%$ in $2050^{7)}$. The prevalence of dementia by age nearly doubles every five years for those aged over 65 years. It was found to be $1.3 \%$ among those aged $65 \sim 69$ years of age, but rapidly increased to $33.9 \%$ in those aged 85 years and above ${ }^{8)}$. For each region, the maximum number of patients with dementia were found in Europe, the United States (80 89 years), and Asia ( $75 \sim 84$ years), thus indicating that the age range of patients with dementia in Asia is decreasing ${ }^{6}$. In Korea, the average dementia counseling center enrollment rate of patients with dementia aged 60 years and above is $52.1 \%^{9)}$. This shows that the age range of patients with dementia is significantly decreasing in Korea.

Dementia is closely related to oral disease, among other causes. In older people with tooth loss, the cognitive function of the brain decreases as the chewing function declines, this may lead to the development of dementia. In fact, dementia and oral health are closely linked because patients with dementia have difficulty in maintaining oral hygiene due to cognitive impairment, thus resulting in poor oral health ${ }^{10)}$. In particular, systemic inflammatory responses caused by periodontitis have been reported to increase the risk of developing Alzheimer's disease, which is the most common cause of dementia ${ }^{11}$. Among the bacteria that cause oral diseases, a common one that causes pneumonia is reported to exist and increase the risk of aspiration pneumonia in patients with dementia ${ }^{12)}$. Therefore, poor oral hygiene is associated with systemic diseases (e.g., pneumonia and cognitive decline), as well as periodontal disease and tooth loss. Cognitive decline is a vicious cycle that also worsens oral health due to improper oral hygiene ${ }^{13}$. In order to prevent dementia, it is necessary to spread the awareness of the importance of the relationship between dementia and oral health so that people can manage their oral health and systemic health simultaneously. Many people have a big fear of dementia, although they are less aware of the relationship between dementia and oral health, and consequently, oral care is often neglected ${ }^{14)}$. Therefore, it is necessary to check the relationship between oral health promotion and dementia prevention behaviors according to the perception of the relationship between dementia and oral health. Most studies to date are about the associations between dementia and oral disease ${ }^{11,12)}$, as well as the relationships between dementia knowledge, dementia anxiety, and dementia prevention behavior in older individuals ${ }^{15,16)}$. In response to the declining age of dementia onset in Korea, it is necessary to compare oral health promotion and dementia prevention behaviors according to the perception level of the relationship between dementia and oral health in people aged 40 years and above, including middle-aged people. This study aimed to identify this relationship in order to use the results as fundamental data for dementia prevention.

\section{Materials and Methods}

\section{Study subjects and method}

This study was conducted between January 10 and 20, 2020 with people aged 40 years and above living in South Korea. The questionnaires were distributed to those who fully understood the purpose of the study and agreed to participate. The questionnaires were self-administered and collected immediately after completion. Those who found it difficult to write independently were interviewed directly and their responses were recorded. The sample size was calculated to be 119 persons by using the $\mathrm{G}^{*}$ Power 3.1 program based on the significance level 0.01 , power $95 \%$, and effect size 0.15 , which is the medium level. Of the 150 respondents who voluntarily agreed to the survey, 140 were chosen, excluding non-responses and uncertain responses.

\section{Study tool}

\section{1) Oral health promotion behavior}

The questionnaire of Shahi et al. ${ }^{17)}$ consisted of six items, and served as reference for the factors of oral health promotion behavior. Each item was measured on a 5-point Likert scale, where a higher score indicated a better intention of oral health promotion behavior. The reliability in this study was Cronbach's $\alpha=0.604$. 


\section{2) Dementia prevention behavior}

Dementia prevention behavior was measured by using the measurement tool developed by Lee et al. ${ }^{18)}$ It consisted of 12 items, including stress management (4 items), disease management (3 items), lifestyle (3 items), and diet (2 items). Each item was measured on a 5-point Likert scale, where a higher score indicated better dementia prevention behavior. The reliability in this study was Cronbach's $\alpha=0.723$.

\section{3) Perception level on the relationship between dementia and oral health}

The questionnaire assessing the perception level of the relationship between dementia and oral health consisted of a total of 10 items, referring to various publications ${ }^{11-17)}$. It included items, such as the number of teeth (1 item), prosthetic treatment (1 item), regular checkups (1 item), oral hygiene ( 2 items), periodontal disease ( 2 items), and chewing ability (3 items). Each item was measured on a 5-point Likert scale, where a higher score indicated a higher perception level of the relationship between dementia and oral health. The reliability in this study was Cronbach's $\alpha=0.830$. Seven points or fewer were classified into the low perception level group based on its cumulative percentage of $52.9 \%$, and eight points or more were classified into the high perception level group.

\section{Statistical analysis}

Data were analyzed using IBM SPSS ver. 25.0 (IBM Corp., Armonk, NY, USA). Frequency analysis was performed for the perception level of the relationship between dementia and oral health, and the demographic characteristics assessed according to the perception level. Independent t-tests were performed for the mean comparison between oral health promotion behavior and dementia prevention behavior. Correlation analysis was performed to assess the relationship between the three variables (oral health promotion and dementia prevention behaviors, the perception level of the relationship between them), and the significance level of the statistical test was 0.05 .

\section{RESULTS}

\section{Perception level of the relationship between dementia and oral health}

Based on the perception level of the relationship between dementia and oral health, the rate of correct answers was high in all items, except for items 3 and 4. In particular, items $8 \sim 10$ showed a high rate of correct answers above 90\% (Table 1).

Table 1. Perception Level of Dementia and Oral Health

\begin{tabular}{|c|c|c|}
\hline Characteristic & Incorrect answer & Correct answer \\
\hline 1. The risk of dementia increases for people with fewer teeth. & $45(32.1)$ & $95(67.9)$ \\
\hline 2. The risk of dementia increases for people without dentures. & $56(40.0)$ & $84(60.0)$ \\
\hline 3. The risk of dementia increases for people who do not brush their teeth regularly. & $79(56.4)$ & $61(43.6)$ \\
\hline $\begin{array}{l}\text { 4. The risk of dementia increases for people who do not have annual regular } \\
\text { dental checkups. }\end{array}$ & $77(55.0)$ & $63(45.0)$ \\
\hline 5. The risk of dementia increases for people with a worsened oral condition. & $55(39.3)$ & $85(60.7)$ \\
\hline 6. The risk of dementia increases for people with worsened periodontal disease. & $48(34.3)$ & $92(65.7)$ \\
\hline $\begin{array}{l}\text { 7. Periodontal bacteria were abnormally found in the brain tissue of people who died } \\
\text { of dementia. }\end{array}$ & $63(37.9)$ & $87(62.1)$ \\
\hline 8. Poor chewing ability can adversely affect brain function due to poor nutrition. & $9(6.4)$ & $131(93.6)$ \\
\hline $\begin{array}{l}\text { 9. Poor chewing ability reduces blood flow oxygen levels in the central nervous } \\
\text { system, which can adversely affect brain function. }\end{array}$ & $9(6.4)$ & $131(93.6)$ \\
\hline $\begin{array}{l}\text { 10. Active chewing can stimulate the nervous system and have a positive effect on } \\
\text { brain function. }\end{array}$ & $14(10.0)$ & $126(90.0)$ \\
\hline Total & $14(10.0)$ & $126(90.0)$ \\
\hline
\end{tabular}

Values are presented as number (\%).

By frequency analysis. 
2. Demographic characteristics according to the perception level of the relationship between dementia and oral health

The result showed that the proportion of the low perception level group was higher in both male and female. In terms of age, high perception level was more prevalent among the age group of 64 years and below whereas low perception was more prevalent among the age group of 65 years and above. Individuals who visited their dentist every three to six months were more prevalent in the high perception level group whereas those who only visited when they were sick were more in the low perception level group (Table 2).

Table 2. Comparative Analysis on the Demographic Characteristics between Dementia and Oral Health Related Perception Level

\begin{tabular}{lcc}
\hline & \multicolumn{2}{c}{$\begin{array}{c}\text { Dementia and oral health related } \\
\text { perception level }\end{array}$} \\
\cline { 2 - 3 } Characteristic & $\begin{array}{c}\text { Low group } \\
(\mathrm{n}=74)\end{array}$ & $\begin{array}{c}\text { High group } \\
(\mathrm{n}=66)\end{array}$ \\
\hline Sex & & \\
Male & $27(52.9)$ & $24(47.1)$ \\
Female & $47(52.8)$ & $42(47.2)$ \\
Age & & \\
$\leq 64$ & $29(47.5)$ & $32(52.5)$ \\
$\geq 65$ & $45(57.0)$ & $34(43.0)$ \\
Dental visit frequency & & \\
$3 \sim 6$ mo & $7(36.8)$ & $12(63.2)$ \\
1 y & $27(57.4)$ & $20(42.6)$ \\
$2 \sim 3$ y & $4(57.1)$ & $3(42.9)$ \\
Only when sick & $36(53.7)$ & $31(46.3)$ \\
\hline
\end{tabular}

Values are presented as number (\%).

By frequency analysis.
3. Oral health promotion behavior according to the perception level on the relationship between dementia and oral health

The result of the oral health promotion behavior analysis shows the high perception level group had a higher oral health promotion behavior with respect to all items on the questionnaire. However, there was a significant difference $(\mathrm{p}<0.05$, Table 3$)$ in all items, except for items 3 and 6.

\section{Dementia prevention behavior according to the perception level on the relationship between dementia and oral health}

The analysis of the dementia prevention behavior shows that the high perception level group showed a higher dementia prevention behavior with respect to all items, except for item 1 . There was a significant difference $(\mathrm{p}<$ 0.05 , Table 4) in items $5,7,8,9$, and 12 .

\section{Associations of the perception level of the relationship among dementia and oral health, oral health promotion behavior, and dementia prevention behavior}

The correlation analysis of the perception level of the relationship between dementia and oral health, oral health promotion behavior, and dementia promotion behavior showed that the three variables all had positive correlations $(\mathrm{p}<0.001$, Table 5).

Table 3. Comparative Analysis of the Oral Health Promotion Behavior between Dementia and Oral Health Related Perception Level

\begin{tabular}{|c|c|c|c|c|}
\hline \multirow{2}{*}{ Characteristic } & \multicolumn{4}{|c|}{ Dementia and oral health related perception level } \\
\hline & Low group $(n=74)$ & High group $(n=66)$ & $\mathrm{t}$ & p-value \\
\hline 1. I visit the dentist on a regular basis for dental checkup. & $3.54 \pm 1.24$ & $4.17 \pm 1.15$ & -3.091 & 0.002 \\
\hline 2. If you have a toothache, you should have it treated immediately. & $4.46 \pm 0.98$ & $4.83 \pm 0.62$ & -2720 & 0.007 \\
\hline 3. I use toothpaste that contains fluoride. & $4.04 \pm 1.33$ & $4.18 \pm 1.23$ & -0.651 & 0.516 \\
\hline 4. I brush my teeth more than three times a day. & $3.84 \pm 1.39$ & $4.53 \pm 0.99$ & -3.422 & 0.001 \\
\hline 5. I carefully brush all of my teeth up and down. & $4.53 \pm 0.88$ & $4.82 \pm 0.46$ & -2.410 & 0.017 \\
\hline 6. I eat my food evenly. & $4.42 \pm 1.03$ & $4.67 \pm 0.73$ & -1.651 & 0.101 \\
\hline Total & $24.82 \pm 3.85$ & $27.20 \pm 3.11$ & -3.977 & $<0.001$ \\
\hline
\end{tabular}

Values are presented as mean \pm standard deviation.

$\mathrm{p}$-values by independent $\mathrm{t}$-test. 
Table 4. Comparative Analysis of the Dementia Prevention Behavior between Dementia and Oral Health Related Perception Level

\begin{tabular}{lrrrr}
\hline \multicolumn{1}{c}{ Characteristic } & \multicolumn{2}{c}{ Dementia and oral health related perception level } \\
\cline { 2 - 5 } & $\begin{array}{c}\text { Low group } \\
(\mathrm{n}=74)\end{array}$ & $\begin{array}{c}\text { High group } \\
(\mathrm{n}=66)\end{array}$ & $\mathrm{t}$ & $\mathrm{p}$-value \\
\hline 1. Do you smoke? & $4.09 \pm 1.35$ & $4.08 \pm 1.41$ & 0.081 & 0.936 \\
2. Do you drink alcohol? & $2.93 \pm 1.53$ & $3.11 \pm 1.48$ & -0.681 & 0.497 \\
3. Do you eat regular meals? & $4.14 \pm 1.11$ & $4.41 \pm 9,84$ & -1.626 & 0.106 \\
4. Do you read newspapers, magazines, etc.? & $3.41 \pm 1.49$ & $3.59 \pm 1.66$ & -0.698 & 0.486 \\
5. Do you eat enough vegetables and fruits? & $4.05 \pm 1.16$ & $4.41 \pm 0.89$ & -2.013 & 0.046 \\
6. Are you maintaining your usual weight? & $4.19 \pm 1.09$ & $4.44 \pm 0.84$ & -1.502 & 0.135 \\
7. Do you usually take good care of your disease? & $4.15 \pm 1.17$ & $4.58 \pm 0.68$ & -2.604 & 0.010 \\
8. Do you exercise for more than 20 min a day on a weekly basis? & $3.77 \pm 1.22$ & $4.45 \pm 0.88$ & -3.829 & $<0.001$ \\
9. Do you usually relieve stress well? & $4.15 \pm 1.16$ & $4.61 \pm 0.63$ & -2.951 & 0.004 \\
10. Are you trying to live happily? & $4.64 \pm 0.80$ & $4.65 \pm 0.79$ & -0.121 & 0.904 \\
11. Do you often do handwork (e.g., embroidery, sewing, etc.)? & $3.07 \pm 1.42$ & $3.55 \pm 1.62$ & -1.848 & 0.067 \\
12. Do you often talk or sing with your friends? & $3.96 \pm 1.14$ & $4.64 \pm 0.78$ & -4.141 & $<0.001$ \\
Total & $46.54 \pm 7.71$ & $50.50 \pm 5.65$ & -3.488 & 0.001 \\
\hline
\end{tabular}

Values are presented as mean \pm standard deviation.

p-values by independent t-test.

Table 5. Relevance among Three Variables

\begin{tabular}{lccc}
\hline \multicolumn{1}{c}{ Characteristic } & $\begin{array}{c}\text { Oral health } \\
\text { promotion behavior }\end{array}$ & $\begin{array}{c}\text { Dementia } \\
\text { prevention behavior }\end{array}$ & $\begin{array}{c}\text { Dementia and oral health } \\
\text { related perception level }\end{array}$ \\
\hline Oral health promotion behavior & 1 & & 1 \\
Dementia prevention behavior & $0.437^{* * *}$ & $0.243^{* * *}$ & 1 \\
Dementia and oral health related perception level & $0.274^{* * *}$ & & 1 \\
\hline
\end{tabular}

p-values by correlation analysis, $* * * \mathrm{p}<0.001$.

\section{DISCUSSION}

Many studies have shown that oral disease is associated with various systemic diseases, such as cardiovascular diseases, diabetes, and respiratory diseases ${ }^{19}$. In recent years, dementia and cognitive impairment, which are degenerative brain diseases emerging as serious problems worldwide, have been reported to be related to oral health. For this reason, oral health care is becoming increasingly important $\mathrm{t}^{20}$. However, many people are unaware of the relationship between dementia and oral health, so it is essential to educate them in order to increase proper awareness and knowledge. In this study, the relationship between dementia and chewing ability showed a high rate of correct answers among the 10 items that investigated the perception level of the relationship between dementia and oral health. The relationship between dementia and periodontal disease, number of teeth, and presence of a denture showed about a 50\% correct answer rate. On the other hand, a higher rate of incorrect answers was shown in the relationship between dementia and brushing and regular oral checkups. This indicates that people recognize the link between oral health deterioration and dementia; however, they rarely recognize that dementia is related to regular oral checkups and brushing, which are behaviors that prevent oral health deterioration. It is necessary for people to recognize this link. The age group of 65 years and above showed a lower perception level of the relationship between dementia and oral health, compared to the age group of 64 years and below, which was consistent with the study of Lee and $\mathrm{Kim}^{14)}$, showing that the knowledge of dementia decreased with age.

In all diseases, prevention (e.g., regular checkups) should be given priority over treatment. Pain in the mouth indicates that the disease is currently progressing, so it is very important to visit a dentist frequently as a preventive 
measure. Patients with short intervals of regular oral checkups had higher levels of perception of the relationship between dementia and oral health, whereas those who visited the dentist only when they needed to had lower levels of perception. As such, it is necessary to increase the perception that oral health is related to dementia so that oral checkups can be performed before the disease progresses.

Oral health promotion behavior is a preventive measure for oral disease. It is very important to analyze oral health promotion behavior in the sense that the spontaneity of the person who practices it plays an important role, and a different one from the disease behavior ${ }^{21)}$. The higher the perception level of the relationship between dementia and oral health, the higher the likelihood of oral health promotion behavior. In particular, large differences between the high and low perception level groups were shown in regular oral checkups and brushing more than three times a day. Tooth brushing is known as the most basic method of removing dental plaque, but is difficult to practice brushing three times a day ${ }^{22)}$. Therefore, we need to drive home the idea that regular oral checkups and proper toothbrushing can help prevent dementia in order to increase oral health promotion behavior. The level of dementia prevention behavior is reported to be higher when awareness and knowledge of the disease is high and the attitude toward dementia is positive ${ }^{23)}$. In this study, the higher the perception level of the relationship between dementia and oral health, the higher the dementia prevention behavior. Moreover, the perception level of the relationship between dementia and oral health, oral health promotion behavior, and dementia prevention behavior were all positively correlated. Since oral health promotion and dementia prevention behaviors are highly related, efforts should be made to promote awareness and knowledge of their relationship. The limitation of this study is that it did not consider various characteristics and knowledge of full-body diseases, drug use, smoking, drinking, socioeconomic characteristics, and oral health that affect oral health promotion and dementia prevention behaviors in middle-aged people. Therefore, an in-depth analysis will have to be made for further research. It would also be necessary to expand the number of people who are socially marginalized, including people with mental illness and disabilities, as well as patients with dementia, to check their relationship with oral health. This study identified the relationship between oral health promotion behavior and dementia prevention behavior according to the perception level of the relationship between dementia and oral health. Based on the above results, it was confirmed that oral health promotion behavior and dementia prevention behavior increased as the perception level of the relationship between dementia and oral health increased. Therefore, in order to prevent dementia, efforts should be made to improve oral health by including oral health education in the education program for dementia.

\section{Notes}

\section{Conflict of interest}

No potential conflict of interest relevant to this article was reported.

\section{Ethical approval}

This study was approved by the institutional review board of the Silla University (IRB No.1041449-201912HR-002).

\section{Author contributions}

Conceptualization: Yu-Rin Kim. Data acquisition: Yu-Rin Kim. Formal analysis: Yu-Rin Kim. Supervision: Yu-Rin Kim. Writing-original draft: Yu-Rin Kim. Writing-review \& editing: Yu-Rin Kim.

\section{ORCID}

Yu-Rin Kim, https://orcid.org/0000-0001-6440-6745

\section{Acknowledgements}

This research did not receive any specific grant from funding agencies in the public, commercial, or not-forprofit sectors.

\section{References}

1. Statistics Korea: Statistical quality control. Retrieved February 1, 2019, from https://www.kostat.go.kr/portal/korea/ 
kor_pi/8/6/2/index.board?bmode=read\&aSeq=379900 $(2020$, January 9).

2. Sunwoo D: A study on research trends and development process of health policy for the elderly. J Korean Geriatr Soc 28: 773-784, 2008.

3. Mayeux R: Epidemiology of neurodegeneration. Annu Rev Neurosci 26: 81-104, 2003. https://doi.org/10.1146/annurev.neuro.26.043002.094919

4. Kang KL: Association between dementia and oral health. J Korean Dent Assoc 56: 218-230, 2018.

5. Ferri CP, Prince M, Brayne C, et al.; Alzheimer's Disease International: Global prevalence of dementia: a Delphi consensus study. Lancet 366: 2112-2117, 2005. https://doi.org/10.1016/S0140-6736(05)67889-0

6. World Health Organization; Alzheimer's Disease International: Dementia: a public health priority. Retrieved February 1, 2019, from https://www.who.int/mental_health/publications/ dementia_report_2012/en/(2012).

7. Statistics Korea: Future population estimation. Retrieved February 1, 2019 from http://www.kostat.go.kr/portal/korea/ kor_nw/1/2/6/index.board(2019, March 28).

8. Ministry of Health and Welfare: 2nd National dementia management comprehensive plan ('13-'15). Retrieved February 1, 2019 from http://www.korea.kr/archive/expDocView. do?docId=32455(2012, July 27).

9. Park MS: Oral care and considerations for dental treatment in dementia patients. J Korean Dent Assoc 56: 231-237, 2018.

10. Riviere GR, Riviere KH, Smith KS: Molecular and immunological evidence of oral Treponema in the human brain and their association with Alzheimer's disease. Oral Microbiol Immunol 17: 113-118, 2002.

https://doi.org/10.1046/j.0902-0055.2001.00100.x

11. Li X, Kolltveit KM, Tronstad L, Olsen I: Systemic diseases caused by oral infection. Clin Microbiol Rev 13: 547-558, 2000. https://doi.org/10.1128/cmr.13.4.547-558.2000

12. Noble JM, Scarmeas N, Papapanou PN: Poor oral health as a chronic, potentially modifiable dementia risk factor: review of the literature. Curr Neurol Neurosci Rep 13: 384, 2013. https://doi.org/10.1007/s11910-013-0384-x

13. Alzheimer's Association: 2014 Alzheimer's disease facts and figures. Alzheimers Dement 10: e47-e92, 2014. https://doi.org/10.1016/j.jalz.2014.02.001
14. Lee KW, Kim AL: Senior citizens relationships between dementia knowledge, dementia anxiety, and dementia prevention behavior in community.J Korea Soc Wellness 13: 357-367, 2018.

https://doi.org/10.21097/ksw.2018.11.13.4.357

15. Go HB, Kim MG, Kim JY, et al.: The relationship between dementia and oral health in some elderly in Daejeon. J Dent Hyg Sci 16: 481-487, 2016. https://doi.org/10.17135/jdhs.2016.16.6.481

16. Choi YK, Kim EJ: Awareness of dementia national responsibility of elders: oral health items. J Dent Hyg Sci 19: 67-75, 2019. https://doi.org/10.17135/jdhs.2019.19.1.67

17. Shahi AK, Chandra S, Kumar S, Sharma S, Prajapati VK, Singh B: Oral health behavior and factors influencing the selection of oral hygiene products among the adolescent tobacco consumers in Jharkhand. J Contemp Dent Pract 20: 1200-1205, 2019. https://doi.org/10.5005/jp-journals-10024-2660

18. Lee YW, Woo SM, Kim OR, Lee SY, Im HB: Relationships between dementia knowledge, attitude, self-efficacy, and preventive behavior among low income middle-aged women. Korean J Adult Nurs 21: 617-627, 2009.

19. Loesche WJ, Lopatin DE: Interactions between periodontal disease, medical diseases and immunity in the older individual. Periodontol 2000 16: 80-105, 1998. https://doi.org/10.1111/j.1600-0757.1998.tb00117.x

20. An ES, Kim MY: Relationship between oral health care behaviors and perceived periodontal disease on hypertension patients. J Dent Hyg Sci 16: 101-109, 2016. https://doi.org/10.17135/jdhs.2016.16.1.101

21. Kim SD: Prevalence of activity limitation in Korea's older adults population. J Korea Contents Assoc 18: 453-460, 2018. https://doi.org/10.5392/JKCA.2018.18.06.453

22. Starke EM, Mwatha A, Ward M, et al.: A comparison of the effects of a powered and manual toothbrush on gingivitis and plaque: a randomized parallel clinical trial. J Clin Dent 30(Spec No A): A24-A29, 2019.

23. Lee JH, Lee EH: Study of the factors affecting behaviors for the promotion of dementia prevention: verifying the mediating effect of an attitude toward dementia. Korean Comp Gov Rev 20: 89-117, 2016. 